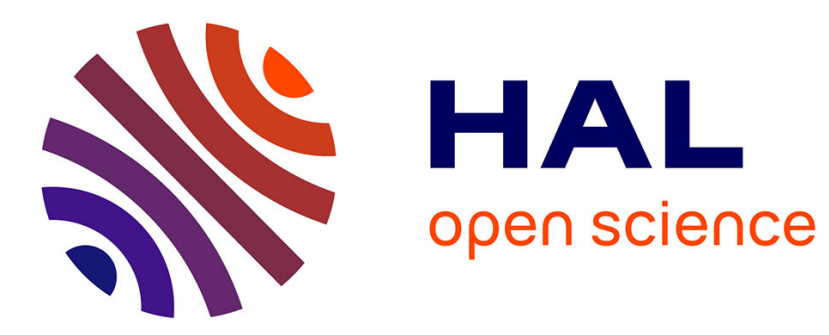

\title{
Imperfect interfaces as asymptotic models of thin curved elastic adhesive interphases
}

Raffaella Rizzoni, Frédéric Lebon

\section{To cite this version:}

Raffaella Rizzoni, Frédéric Lebon. Imperfect interfaces as asymptotic models of thin curved elastic adhesive interphases. Mechanics Research Communications, 2013, 51, pp.39- 50. 10.1016/j.mechrescom.2013.04.008 . hal-00833562

\section{HAL Id: hal-00833562 \\ https://hal.science/hal-00833562}

Submitted on 20 May 2018

HAL is a multi-disciplinary open access archive for the deposit and dissemination of scientific research documents, whether they are published or not. The documents may come from teaching and research institutions in France or abroad, or from public or private research centers.
L'archive ouverte pluridisciplinaire HAL, est destinée au dépôt et à la diffusion de documents scientifiques de niveau recherche, publiés ou non, émanant des établissements d'enseignement et de recherche français ou étrangers, des laboratoires publics ou privés. 


\title{
Imperfect interfaces as asymptotic models of thin curved elastic adhesive interphases
}

\author{
Raffaella Rizzoni ${ }^{\mathrm{b}, *}$, Frédéric Lebon ${ }^{\mathrm{a}}$ \\ a CNRS, Laboratoire de Mécanique et d'Acoustique, Université Aix-Marseille, 31 Chemin Joseph-Aiguier, 13402 Marseille Cedex 20, France \\ ${ }^{\mathrm{b}}$ Dipartimento di Ingegneria, Università of Ferrara, Via Saragat 1, 44122 Ferrara, Italy
}

\begin{abstract}
We obtain a limit model for a thin curved anisotropic interphase adherent to two elastic media. Our method is based on asymptotic expansions and energy minimization procedures. The model of perfect interface is obtained at the first order, while an imperfect interface model is obtained at the next order. The conditions of imperfect contact, given in a parallel orthogonal curvilinear coordinate system, involve the interphase material properties, the first order displacement and traction vectors, and their derivatives. An example of implementation of the imperfect interface condition is given for a composite sphere assemblage.
\end{abstract}

\section{Introduction}

During the last few years gluing techniques have become usual in engineering structural assembly and they are now frequent in aeronautics industry, where the use of composite material is necessary to lighten structures. These techniques have to be taken into account in structural modeling, in order to develop implementable predictive models in computational structural analysis softwares.

The thickness of the glue is usually very small when compared with the characteristic dimensions of the structure. A classical modeling approach is to consider the thickness as a small parameter and to study the limit problem when this parameter tends to zero. Traditionally, the stiffness of the glue is assumed to be smaller than those of the adherents. Many authors focused on the assumption of soft adhesively bonded joining, with fundamental contributions given in Klarbring (1991) and Klarbring and Movchan (1998) and in works addressing the assembly of elastic plates (Geymonat and Krasucki, 1997; Zaittouni et al., 2002), the limit behavior of soft thin interphases (Licht, 1993; Licht and Michaille, 1996, 1997; Ould-Khaoua et al., 1996; Ganghoffer et al., 1997; Lebon et al., 1997, 2004; Geymonat et al., 1999; Lenci, 2000; Lebon and Ronel-Idrissi, 2004; Lebon and Rizzoni, 2008; Cognard et al., 2008; Schmidt, 2008; Pelissou and Lebon, 2009; Rekik and Lebon, 2012; Monchiet and Bonnet, 2010), and, more recently, the dynamics of laminated beams (Serpilli and Lenci, 2012).

On the other hand, the ratio between the stiffness of the glue and the stiffness of the adherents can be larger than $1 / 50$, about $1 / 35$ for two aluminum plates joined by an epoxy glue. Thus, it is interesting to consider the case in which the elastic moduli of the glue and the adherents are comparable. This case has been studied in Caillerie (1980) and Abdelmoula et al. (1998) and in some recent works focusing on interphase modeling in elasticity (Benveniste and Miloh, 2001; Hashin, 2002; Benveniste, 2006; Lebon and Ronel, 2007; Serpilli and Lenci, 2008; Benveniste and Berdichevsky, 2010; Lebon and Rizzoni, 2010, 2011; Lebon and Zaittouni, 2010; Rizzoni and Lebon, 2012) and on structural interfaces (Bigoni and Movchan, 2002; Bertoldi et al., 2007a,b).

In particular, in Lebon and Rizzoni (2011) a two-level model of a flat imperfect interface was derived using an energy asymptotic method. At the first level, the adhesive is replaced by a model of perfect interface, for which the stress and the displacement vectors are continuous. At the second level, the jumps in the displacements and in the stress vector along the interface are related to the interphase material properties and to the displacement and stress fields obtained at the first level.

In the present paper, we extend the results obtained in Lebon and Rizzoni (2011) to the case of a curved, thin, linear elastic, anisotropic and homogeneous interphase joining two elastic adherents. In Section 2, we introduce the general notations and the three-dimensional

\footnotetext{
* Corresponding author. Tel.: +39 0532 974959; fax: +39 0532974959.

E-mail addresses: raffaella.rizzoni@unife.it, rzzrfl@unife.it (R. Rizzoni), lebon@lma.cnrs-mrs.fr (F. Lebon).
} 
equilibrium problem posed in a variational form. In Section 3, we obtain two different types of interface condition, by using a method based on asymptotic expansions and energy minimization procedures. These conditions extend to the curvilinear case the interface conditions obtained in Lebon and Rizzoni (2011) for a straight interface.

As found in Lebon and Rizzoni (2011), the first order term of the expansion yields the model of perfect interface. The second order term yields an imperfect interface model simulating the presence of the interphase. We interpret this term as a correction of the leading solution corresponding to the perfect interface model. The conditions of imperfect contact, obtained in a parallel orthogonal curvilinear coordinate system for a general anisotropic homogeneous interphase material, are specialized in Section 4 to the case of isotropic interphase. Section 5 is devoted to the implementation of the imperfect interface conditions and an example of a composite spheres assemblage is given in Section 6.

\section{Formulation of the three-dimensional equilibrium problem}

Let us consider a thin curved adhesive interphase $B^{\varepsilon} \in \mathbb{R}^{3}$ of constant thickness $\varepsilon$ bonding two adherents occupying the regions $\Omega_{+}^{\varepsilon}, \Omega_{-}^{\varepsilon} \in$ $\mathbb{R}^{3}$. Two parallel interfaces, denoted $S_{+}^{\varepsilon}$ and $S_{-}^{\varepsilon}$, separate $\Omega_{+}^{\varepsilon}$ and $\Omega_{-}^{\varepsilon}$ from $B^{\varepsilon}$, respectively. Let $S$ denote the middle surface between $S_{+}^{\varepsilon}$ and $S_{-}^{\varepsilon}$. These interfaces are assumed to be perfect with the usual assumption that the displacement vector, $u^{\varepsilon}$, and the stress vector, $\sigma^{\varepsilon} n$, are continuous on $S_{ \pm}^{\varepsilon}$. Our goal is to substitute the thin interphase $B^{\varepsilon}$ with the surface $S$, geometric limit of the interphase as $\varepsilon \rightarrow 0^{+}$, and to determine the imperfect interface conditions on the displacement and tractions across $S$ which are equivalent to the three phase configuration with perfect interface conditions. The three regions are assumed to be anisotropic, homogeneous and linear elastic. We take $\mathbf{a}_{ \pm}$to denote the elasticity tensors of the materials occupying the regions $\Omega_{ \pm}^{\varepsilon}$, and $\mathbf{b}$ the elasticity tensor of the interphase material. The elasticity tensors are assumed to satisfy the following assumptions:

$$
\left\{\begin{array}{l}
\mathbf{a}_{ \pm} \in L^{\infty}\left(\Omega_{+}^{\varepsilon} \cup \Omega_{-}^{\varepsilon}\right), \quad \mathbf{b} \in L^{\infty}\left(B^{\varepsilon}\right), \\
\exists \eta_{ \pm}, \eta>0: \mathbf{a}_{ \pm}(e) \cdot(e) \geq \eta_{ \pm}|e|^{2}, \mathbf{b}(e) \cdot(e) \geq \eta|e|^{2}, \quad \forall e: e=e^{T},
\end{array}\right.
$$

and the standard assumptions of major and minor symmetries. After taking $e$ to denote the strain tensor

$$
e\left(u^{\varepsilon}\right)=\frac{1}{2}\left(\nabla u^{\varepsilon}+\left(\nabla u^{\varepsilon}\right)^{T}\right)
$$

linear elasticity gives the Cauchy stress tensor $\sigma^{\varepsilon}$ as follows:

$$
\begin{aligned}
\sigma^{\varepsilon} & =\mathbf{a}_{ \pm}(e) \text { in } \Omega_{ \pm}^{\varepsilon}, \\
\sigma^{\varepsilon} & =\mathbf{b}(e) \text { in } B^{\varepsilon} .
\end{aligned}
$$

A body force density $f \in L^{2}\left(\Omega_{+}^{\varepsilon} \cup \Omega_{-}^{\varepsilon}\right)^{3}$ is assumed to be applied to $\left(\Omega_{+}^{\varepsilon} \cup \Omega_{-}^{\varepsilon}\right)$ and a surface force density $g$ to $\Gamma_{g}^{\varepsilon} \subset\left(\partial \Omega_{+}^{\varepsilon} \cup \partial \Omega_{-}^{\varepsilon}\right)$, with $g \in L^{2}\left(\Gamma_{g}^{\varepsilon}\right)^{3}$. Homogeneous boundary conditions are prescribed on $\Gamma_{u}^{\varepsilon}:=\left(\partial \Omega_{+}^{\varepsilon} \cup \partial \Omega_{-}^{\varepsilon}\right) \backslash \Gamma_{g}^{\varepsilon}$ :

$$
u^{\varepsilon}=0 \text { on } \Gamma_{u}^{\varepsilon} \text {. }
$$

The equilibrium configurations of the three-phase composite body are the minimizers of the total energy

$$
E^{\varepsilon}(u)=\int_{\Omega_{ \pm}^{\varepsilon}}\left(\frac{1}{2} \mathbf{a}_{ \pm}\left(e\left(u^{\varepsilon}\right)\right) \cdot e\left(u^{\varepsilon}\right)-f \cdot u^{\varepsilon}\right) d V-\int_{\Gamma_{g}^{\varepsilon}} g \cdot u^{\varepsilon} d A+\int_{B^{\varepsilon}} \frac{1}{2} \mathbf{b}\left(e\left(u^{\varepsilon}\right)\right) \cdot e\left(u^{\varepsilon}\right) d V
$$

in the space of kinematically admissible displacements

$$
V^{\varepsilon}=\left\{u \in H\left(\Omega^{\varepsilon} ; R^{3}\right): u=0 \text { on } \Gamma_{u}^{\varepsilon}\right\},
$$

where $H\left(\Omega^{\varepsilon} ; R^{3}\right)$ is the space of the vector-valued functions on the set $\Omega^{\varepsilon}:=\left(\Omega_{+}^{\varepsilon} \cup \Omega_{-}^{\varepsilon} \cup B^{\varepsilon} \cup S_{+}^{\varepsilon} \cup S_{-}^{\varepsilon}\right)$, which are continuous and differentiable as many times as necessary. In view of the above regularity assumptions on $\mathbf{a}_{ \pm}, \mathbf{b}, f$, and $g$, the existence of a unique minimizer $u^{\varepsilon}$ in $V^{\varepsilon}$ is ensured (Ciarlet, 1988, Theorem 6.3).

\section{Thin interphase asymptotic analysis}

In this section, we follow the asymptotic method based on the energy minimization and introduced in Lebon and Rizzoni (2011). The first step of the method consists in reformulating the equilibrium problem for a rescaled interphase domain made independent of $\varepsilon$ via a change of variables. To do so, it is convenient to introduce a curvilinear orthogonal system $\left(\xi_{1}, \xi_{2}, \xi_{3}\right)$ in $\Omega^{\varepsilon}$, where $\xi_{1}$ and $\xi_{2}$ are two coordinate lines on the surface $S$ and $\xi_{3}$ is the coordinate line along the normal to the surface (Fig. 1). We assume that $\xi_{3}$ takes the values $\xi_{30}-\varepsilon / 2, \xi_{30}$, and $\xi_{30}+\varepsilon / 2$, on $S_{-}^{\varepsilon}, S$, and $S_{+}^{\varepsilon}$, respectively. Let $\left(\hat{\mathbf{e}}_{1}, \hat{\mathbf{e}}_{2}, \hat{\mathbf{e}}_{3}\right)$ denote the local triad of the curvilinear coordinate system. The metric coefficients of this orthogonal parallel curvilinear coordinate system are denoted by $h_{1}^{\varepsilon}, h_{2}^{\varepsilon}, h_{3}^{\varepsilon}$, with $h_{3}^{\varepsilon}=1$.

The displacement gradient in the orthogonal parallel curvilinear system can be written in the form:

$$
\nabla u^{\varepsilon}=\left(v_{1}^{\varepsilon}\left|v_{2}^{\varepsilon}\right| u_{, 3}^{\varepsilon}\right)
$$

where a comma is used to denote partial differentiation with respect to $\xi_{1}, \xi_{2}, \xi_{3}$, the notation $(a|b| c)$ indicates the matrix whose columns are the vectors $a, b, c$, and

$$
v_{\alpha}^{\varepsilon}:=u_{, \alpha}^{\varepsilon}\left(h_{\alpha}^{\varepsilon}\right)^{-1}+\eta_{\alpha}^{\varepsilon}\left(u^{\varepsilon}\right), \quad \alpha=1,2,
$$




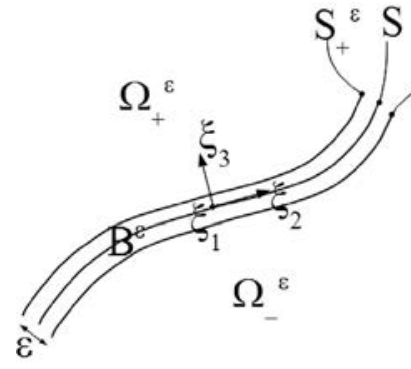

(a)

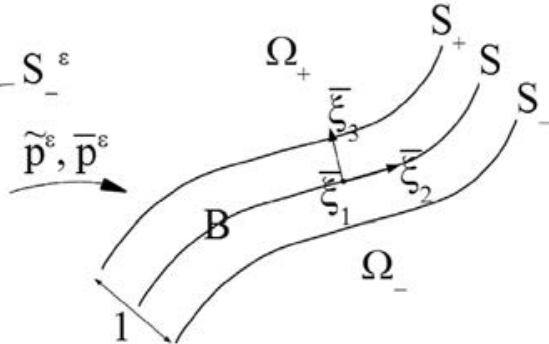

(b)

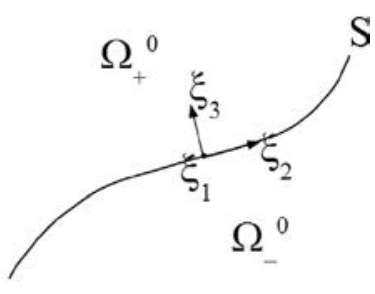

(c)

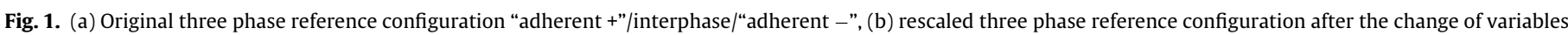
and (c) limit two phase reference configuration.

with

$$
\begin{aligned}
\eta_{1}^{\varepsilon}:=\frac{h_{1,2}^{\varepsilon}}{h_{1}^{\varepsilon} h_{2}^{\varepsilon}}\left(\hat{\mathbf{e}}_{1} \otimes \hat{\mathbf{e}}_{2}-\hat{\mathbf{e}}_{2} \otimes \hat{\mathbf{e}}_{1}\right)+\frac{h_{1,3}^{\varepsilon}}{h_{1}^{\varepsilon}}\left(\hat{\mathbf{e}}_{1} \otimes \hat{\mathbf{e}}_{3}-\hat{\mathbf{e}}_{3} \otimes \hat{\mathbf{e}}_{1}\right), \\
\eta_{2}^{\varepsilon}:=\frac{h_{2,1}^{\varepsilon}}{h_{1}^{\varepsilon} h_{2}^{\varepsilon}}\left(\hat{\mathbf{e}}_{2} \otimes \hat{\mathbf{e}}_{1}-\hat{\mathbf{e}}_{1} \otimes \hat{\mathbf{e}}_{2}\right)+\frac{h_{2,3}^{\varepsilon}}{h_{2}^{\varepsilon}}\left(\hat{\mathbf{e}}_{2} \otimes \hat{\mathbf{e}}_{3}-\hat{\mathbf{e}}_{3} \otimes \hat{\mathbf{e}}_{2}\right) .
\end{aligned}
$$

We now introduce the following change of variables:

$$
\begin{aligned}
& \left(\hat{\bar{\xi}}, \bar{\xi}_{3}\right):=\bar{p}\left(\hat{\xi}, \xi_{3}\right):=\left(\hat{\xi}, \xi_{30}+\varepsilon^{-1}\left(\xi_{3}-\xi_{30}\right)\right) \text { in } B^{\varepsilon} \\
& \left(\hat{\tilde{\xi}}, \tilde{\xi}_{3}\right)=\tilde{p}\left(\hat{\xi}, \xi_{3}\right):=\left(\hat{\xi}, \xi_{3}\right) \pm\left(\frac{\varepsilon}{2} \mp \frac{1}{2}\right) \hat{\mathbf{e}}_{3} \text { in } \Omega_{ \pm}^{\varepsilon},
\end{aligned}
$$

where $\hat{\bar{\xi}}:=\left(\bar{\xi}_{1}, \bar{\xi}_{2}\right)$ and $\hat{\xi}:=\left(\xi_{1}, \xi_{2}\right)$ are the surface coordinates. We take $\Omega_{ \pm}, B, S_{ \pm}, \Gamma_{g}$ and $\Gamma_{u}$ to denote the domains corresponding to $\Omega_{ \pm}^{\varepsilon}, B^{\varepsilon}, S_{ \pm}^{\varepsilon}, \Gamma_{g}^{\varepsilon}$ and $\Gamma_{u}^{\varepsilon}$, respectively, after the change of variables (Fig. 1 ).

Let $\tilde{f}:=f \circ \tilde{p}^{-1}$ and $\tilde{g}:=g \circ \tilde{p}^{-1}$ denote the rescaled external forces.

Let $\tilde{u}_{+}^{\varepsilon}=u^{\varepsilon} \circ \tilde{p}^{-1}$ denote the displacement vector field from the adherents adjacent to the rescaled interphase and let $\bar{u}^{\varepsilon}=u^{\varepsilon} \circ \bar{p}^{-1}$ denote the displacement vector field from the rescaled interphase. In view of the condition of perfect interfaces between the adherents and the rescaled interphase, we have that $\tilde{u}_{ \pm}^{\varepsilon}=\bar{u}^{\varepsilon}$ on $S_{ \pm}$.

Finally, let $\bar{h}_{i}^{\varepsilon}:=h_{i}^{\varepsilon} \circ \bar{p}^{-1}$ and $\tilde{h}_{i}^{\varepsilon}:=h_{i}^{\varepsilon} \circ \tilde{p}^{-1}, i=1,2,3$, denote the rescaled metric coefficients of the curvilinear coordinates system. Note that $\bar{h}_{3}=1=\tilde{h}_{3}$.

Within these assumptions and in view of relations (8)-(11), the rescaled energy of the composite takes the following form:

$$
\begin{aligned}
\mathcal{E}^{\varepsilon}\left(\tilde{u}_{ \pm}^{\varepsilon}, \bar{u}^{\varepsilon}\right):= & \int_{\Omega_{ \pm}}\left(\frac{1}{2} \mathbf{a}_{ \pm}\left(e\left(\tilde{u}_{ \pm}^{\varepsilon}\right)\right) \cdot e\left(\tilde{u}_{ \pm}^{\varepsilon}\right)-\tilde{f} \cdot \tilde{u}_{ \pm}^{\varepsilon}\right) \tilde{h}_{1}^{\varepsilon} \tilde{h}_{2}^{\varepsilon} d V_{\tilde{\xi}}-\int_{\Gamma_{g}}\left(\tilde{g} \cdot \tilde{u}_{ \pm}^{\varepsilon}\right) \tilde{h}_{s}^{\varepsilon} d A_{\tilde{\xi}} \\
& +\int_{B} \frac{1}{2}\left(\frac{1}{\varepsilon} \mathbf{B}^{33}\left(\bar{u}_{, 3}^{\varepsilon}\right) \cdot\left(\bar{u}_{, 3}^{\varepsilon}\right)+2 \mathbf{B}^{\alpha 3}\left(\bar{v}_{\alpha}^{\varepsilon}\right) \cdot\left(\bar{u}_{, 3}^{\varepsilon}\right)+\varepsilon \mathbf{B}^{\alpha \beta}\left(\bar{v}_{\alpha}^{\varepsilon}\right) \cdot\left(\bar{v}_{\beta}^{\varepsilon}\right)\right) \bar{h}_{1}^{\varepsilon} \bar{h}_{2}^{\varepsilon} d V_{\bar{\xi}},
\end{aligned}
$$

where summation convention for repeated indices is used, the indices $\alpha, \beta$ take the values $1,2, \tilde{h}_{s}^{\varepsilon}$ is the Jacobian of the change of variables (12) and (13) in $\Gamma_{g}$, and $\mathbf{B}^{i j}, i, j=1,2,3$ are the matrices whose components are defined by the relations

$$
\mathbf{B}_{h k}^{i j}:=\mathbf{b}_{h i k j} \text {. }
$$

Note that the symmetry properties of $\mathbf{b}$ imply that $\left(\mathbf{B}^{i j}\right)^{T}=\mathbf{B}^{i j}$.

The equilibrium problem of the rescaled three phase configuration can now be formulated as follows: find the pair $\left(\tilde{u}_{ \pm}^{\varepsilon}, \bar{u}^{\varepsilon}\right)$ minimizing the energy (14) in the set of displacements

$$
V=\left\{\left(\tilde{u}_{ \pm}, \bar{u}\right) \in H\left(\Omega_{ \pm} ; R^{3}\right) \times H\left(\bar{B} ; R^{3}\right): \tilde{u}_{ \pm}=\bar{u} \text { on } S_{ \pm}, \quad \tilde{u}_{ \pm}=0 \text { on } \Gamma_{u}\right\}
$$

The asymptotic method that we use in this paper is based upon the existence of the following expansions for the rescaled displacement vector fields $\tilde{u}_{ \pm}^{\varepsilon}, \bar{u}^{\varepsilon}$, for the rescaled metric coefficients, $\tilde{h}_{i}^{\varepsilon}, \bar{h}_{i}^{\varepsilon}, i=1,2,3$, and for the Jacobian $\tilde{h}_{s}^{\varepsilon}$ :

$$
\begin{aligned}
& \tilde{u}_{ \pm}^{\varepsilon}=\tilde{u}_{ \pm}^{0}+\varepsilon \tilde{u}_{ \pm}^{1}+\varepsilon^{2} \tilde{u}_{ \pm}^{2}+o\left(\varepsilon^{2}\right), \\
& \bar{u}^{\varepsilon}=\bar{u}^{0}+\varepsilon \bar{u}^{1}+\varepsilon^{2} \bar{u}^{2}+o\left(\varepsilon^{2}\right), \\
& \tilde{h}_{i}^{\varepsilon}=\tilde{h}_{i}^{0}+\varepsilon \tilde{h}_{i}^{1}+\varepsilon^{2} \tilde{h}_{i}^{2}+o\left(\varepsilon^{2}\right), \quad i=1,2,3,
\end{aligned}
$$




$$
\begin{aligned}
& \bar{h}_{i}^{\varepsilon}=\bar{h}_{i}^{0}+\varepsilon \bar{h}_{i}^{1}+\varepsilon^{2} \bar{h}_{i}^{2}+o\left(\varepsilon^{2}\right), \quad i=1,2,3, \\
& \tilde{h}_{s}^{\varepsilon}=\tilde{h}_{s}^{0}+\varepsilon \tilde{h}_{s}^{1}+\varepsilon^{2} \tilde{h}_{s}^{2}+o\left(\varepsilon^{2}\right) .
\end{aligned}
$$

Substituting these expansions into the rescaled energy (14), we obtain

$$
\mathcal{E}^{\varepsilon}\left(\tilde{u}_{ \pm}, \bar{u}\right)=\frac{1}{\varepsilon} \mathcal{E}^{-1}\left(\bar{u}^{0}\right)+\mathcal{E}^{0}\left(\tilde{u}_{ \pm}^{0}, \bar{u}^{0}, \bar{u}^{1}\right)+\varepsilon \mathcal{E}^{1}\left(\tilde{u}_{ \pm}^{0}, \tilde{u}_{ \pm}^{1}, \bar{u}^{0}, \bar{u}^{1}, \bar{u}^{2}\right)+\varepsilon^{2} \mathcal{E}^{2}\left(\tilde{u}_{ \pm}^{0}, \tilde{u}_{ \pm}^{1}, \tilde{u}_{ \pm}^{2}, \bar{u}^{0}, \bar{u}^{1}, \bar{u}^{2}, \bar{u}^{3}\right)+o\left(\varepsilon^{2}\right),
$$

where

$$
\begin{aligned}
& \mathcal{E}^{-1}\left(\bar{u}^{0}\right):=\int_{B} \bar{w}^{-1} \bar{H}^{0} d V_{\bar{\xi}}, \\
& \mathcal{E}^{0}\left(\tilde{u}_{ \pm}^{0}, \bar{u}^{0}, \bar{u}^{1}\right):=\int_{\Omega_{ \pm}} \tilde{w}^{0} \tilde{H}^{0} d V_{\tilde{\xi}}-\int_{\Gamma_{g}} \tilde{w}_{s}^{0} \tilde{h}_{s}^{0} d A_{\tilde{\xi}}+\int_{B}\left(\bar{w}^{-1} \bar{H}^{1}+\bar{w}^{0} \bar{H}^{0}\right) d V_{\bar{\xi}}, \\
& \mathcal{E}^{1}\left(\tilde{u}_{ \pm}^{0}, \tilde{u}_{ \pm}^{1}, \bar{u}^{0}, \bar{u}^{1}, \bar{u}^{2}\right):=\int_{\Omega_{ \pm}}\left(\tilde{w}^{0} \tilde{H}^{1}+\tilde{w}^{1} \tilde{H}^{0}\right) d V_{\tilde{\xi}}-\int_{\Gamma_{g}}\left(\tilde{w}_{s}^{0} \tilde{h}_{s}^{1}+\tilde{w}_{s}^{1} \tilde{h}_{s}^{0}\right) d A_{\tilde{\xi}}+\int_{B}\left(\bar{w}^{-1} \bar{H}^{2}+\bar{w}^{0} \bar{H}^{1}+\bar{w}^{1} \bar{H}^{0}\right) d V_{\bar{\xi}}, \\
& \mathcal{E}^{2}\left(\tilde{u}_{ \pm}^{0}, \tilde{u}_{ \pm}^{1}, \tilde{u}_{ \pm}^{2}, \bar{u}^{0}, \bar{u}^{1}, \bar{u}^{2}, \bar{u}^{3}\right):= \\
& \quad \int_{\Omega_{ \pm}}\left(\tilde{w}^{0} \tilde{H}^{2}+\tilde{w}^{1} \tilde{H}^{1}+\tilde{w}^{2} \tilde{H}^{0}\right) d V_{\tilde{\xi}}-\int_{\Gamma_{g}}\left(\tilde{w}_{s}^{0} \tilde{h}_{s}^{2}+\tilde{w}_{s}^{1} \tilde{h}_{s}^{1}+\tilde{w}_{s}^{2} \tilde{h}_{s}^{0}\right) d A_{\tilde{\xi}} \\
& \quad+\int_{B}\left(\bar{w}^{-1} \bar{H}^{3}+\bar{w}^{0} \bar{H}^{2}+\bar{w}^{1} \bar{H}^{1}+\bar{w}^{2} \bar{H}^{0}\right) d V_{\bar{\xi}},
\end{aligned}
$$

being

$$
\begin{aligned}
& \tilde{w}^{0}\left(\tilde{u}_{ \pm}^{0}\right):=\frac{1}{2} \mathbf{a}_{ \pm}\left(e\left(\tilde{u}_{ \pm}^{0}\right)\right) \cdot e\left(\tilde{u}_{ \pm}^{0}\right)-\tilde{f} \cdot \tilde{u}_{ \pm}^{0} \\
& \tilde{w}^{1}\left(\tilde{u}_{ \pm}^{0}, \tilde{u}_{ \pm}^{1}\right):=\mathbf{a}_{ \pm}\left(e\left(\tilde{u}_{ \pm}^{0}\right)\right) \cdot e\left(\tilde{u}_{ \pm}^{1}\right)-\tilde{f} \cdot \tilde{u}_{ \pm}^{1} \\
& \tilde{w}^{2}\left(\tilde{u}_{ \pm}^{0}, \tilde{u}_{ \pm}^{1}, \tilde{u}_{ \pm}^{2}\right):=\frac{1}{2} \mathbf{a}_{ \pm}\left(e\left(\tilde{u}_{ \pm}^{1}\right)\right) \cdot e\left(\tilde{u}_{ \pm}^{1}\right)+\mathbf{a}_{ \pm}\left(e\left(\tilde{u}_{ \pm}^{0}\right)\right) \cdot e\left(\tilde{u}_{ \pm}^{2}\right)-\tilde{f} \cdot \tilde{u}_{ \pm}^{2} \\
& \tilde{w}_{s}^{i}\left(\tilde{u}_{ \pm}^{i}\right):=-\tilde{g} \cdot \tilde{u}_{ \pm}^{i}, \quad i=0,1,2 \\
& \bar{w}^{-1}\left(\bar{u}^{0}\right):=\frac{1}{2} \mathbf{B}^{33}\left(\bar{u}_{, 3}^{0}\right) \cdot\left(\bar{u}_{, 3}^{0}\right) \\
& \bar{w}^{0}\left(\bar{u}^{0}, \bar{u}^{1}\right):=\mathbf{B}^{33}\left(\bar{u}_{, 3}^{0}\right) \cdot\left(\bar{u}_{, 3}^{1}\right)+\frac{1}{2} \mathbf{B}^{\alpha 3}\left(\bar{v}_{\alpha}^{0}\right) \cdot\left(\bar{u}_{, 3}^{0}\right), \\
& \bar{w}^{1}\left(\bar{u}^{0}, \bar{u}^{1}, \bar{u}^{2}\right):=\mathbf{B}^{33}\left(\bar{u}_{, 3}^{0}\right) \cdot\left(\bar{u}_{, 3}^{2}\right)+\frac{1}{2} \mathbf{B}^{33}\left(\bar{u}_{, 3}^{1}\right) \cdot\left(\bar{u}_{, 3}^{1}\right)+\frac{1}{2} \mathbf{B}^{\alpha 3}\left(\bar{v}_{\alpha}^{0}\right) \cdot\left(\bar{u}_{, 3}^{1}\right)+\frac{1}{2} \mathbf{B}^{\alpha 3}\left(\bar{v}_{\alpha}^{1}\right) \cdot\left(\bar{u}_{, 3}^{0}\right)+\frac{1}{2} \mathbf{B}^{\alpha \beta}\left(\bar{v}_{\alpha}^{0}\right) \cdot\left(\bar{v}_{\beta}^{0}\right) \\
& \bar{w}^{2}\left(\bar{u}^{0}, \bar{u}^{1}, \bar{u}^{2}, \bar{u}^{3}\right):=\mathbf{B}^{33}\left(\bar{u}_{, 3}^{0}\right) \cdot\left(\bar{u}_{, 3}^{3}\right)+\mathbf{B}^{33}\left(\bar{u}_{, 3}^{1}\right) \cdot\left(\bar{u}_{, 3}^{2}\right)+\frac{1}{2} \mathbf{B}^{\alpha 3}\left(\bar{v}_{\alpha}^{0}\right) \cdot\left(\bar{u}_{, 3}^{2}\right)+\frac{1}{2} \mathbf{B}^{\alpha 3}\left(\bar{v}_{\alpha}^{1}\right) \cdot\left(\bar{u}_{, 3}^{1}\right)+\frac{1}{2} \mathbf{B}^{\alpha 3}\left(\bar{v}_{\alpha}^{2}\right) \cdot\left(\bar{u}_{, 3}^{0}\right)+\mathbf{B}^{\alpha \beta}\left(\bar{v}_{\alpha}^{0}\right) \cdot\left(\bar{v}_{\beta}^{1}\right)
\end{aligned}
$$

In (23)-(26) the following quantities were also defined:

$$
\begin{aligned}
& \tilde{H}^{0}:=\tilde{h}_{1}^{0} \tilde{h}_{2}^{0}, \\
& \tilde{H}^{1}:=\tilde{h}_{1}^{0} \tilde{h}_{2}^{1}+\tilde{h}_{1}^{1} \tilde{h}_{2}^{0}, \\
& \tilde{H}^{2}:=\tilde{h}_{1}^{0} \tilde{h}_{2}^{2}+\tilde{h}_{1}^{1} \tilde{h}_{2}^{2}+\tilde{h}_{1}^{2} \tilde{h}_{2}^{1}+\tilde{h}_{1}^{3} \tilde{h}_{2}^{0},
\end{aligned}
$$

and analogous definitions were introduced for the quantities $\bar{H}^{i}, i=0,1,2$.

In the next subsections, we obtain the conditions satisfied by the stationary points of the energies $\mathcal{E}^{-1}, \mathcal{E}^{0}, \mathcal{E}^{1}$ and $\mathcal{E}^{2}$. These conditions identify the relations between $\bar{u}^{0}, \bar{u}^{1}, \bar{u}^{2}, \ldots$ and the corresponding stress vectors arising from $\tilde{u}_{ \pm}^{0}, \tilde{u}_{ \pm}^{1}, \tilde{u}_{ \pm}^{2}, \ldots$ at the interfaces $S_{+}, S_{-}$.

4 


\subsection{Stationary points of $\mathcal{E}^{-1}$}

We minimize the energy $\mathcal{E}^{-1}$ in the class of displacements $\bar{u}^{0} \in H\left(\bar{B} ; R^{3}\right)$. Since $\mathbf{b}$ is a positive definite tensor, the matrix $\mathbf{B}^{33}$ is also positive definite and thus the energy $\mathcal{E}^{-1}$ is non negative. The stationarity of $\mathcal{E}^{-1}$ with respect to $\bar{u}^{0}$ gives the condition

$$
\bar{u}_{, 3}^{0}=0 \text {, a.e. in } B
$$

i.e., minimizers are independent of the coordinate $\bar{\xi}_{3}$ along the interphase thickness. Based on this result and on the initial assumption of perfect contact between the rescaled interphase and adherents, we obtain the following condition:

$$
\tilde{u}^{0}\left(\hat{\bar{\xi}},\left(\xi_{30}+\frac{1}{2}\right)^{+}\right)=\tilde{u}^{0}\left(\hat{\bar{\xi}},\left(\xi_{30}-\frac{1}{2}\right)^{-}\right), \quad \hat{\bar{\xi}} \in S
$$

If we introduce the following definition of "jump" of a function $\mathfrak{f}: \Omega_{+} \cup \Omega_{-} \mapsto R^{3}$ across the rescaled interphase

$$
[\mathfrak{f}](\hat{\bar{\xi}}):=\mathfrak{f}\left(\hat{\bar{\xi}},\left(\xi_{30}+\frac{1}{2}\right)^{+}\right)-\mathfrak{f}\left(\hat{\bar{\xi}},\left(\xi_{30}-\frac{1}{2}\right)^{-}\right), \quad \hat{\bar{\xi}} \in S
$$

then condition (39) can be rephrased as follows:

$$
\left[\tilde{u}^{0}\right]=0 \text { on } S \text {. }
$$

\subsection{Stationary points of $\mathcal{E}^{0}$}

In view of (38), the energy $\mathcal{E}^{0}$ simplifies as follows:

$$
\mathcal{E}^{0}=\int_{\Omega_{ \pm}}\left(\frac{1}{2} \mathbf{a}_{ \pm}\left(e\left(\tilde{u}_{ \pm}^{0}\right)\right) \cdot e\left(\tilde{u}_{ \pm}^{0}\right)-\tilde{f} \cdot \tilde{u}_{ \pm}^{0}\right) \tilde{H}^{0} d V_{\tilde{\xi}}-\int_{\Gamma_{g}}\left(\tilde{g} \cdot \tilde{u}_{ \pm}^{0}\right) \tilde{h}_{s}^{0} d A_{\tilde{\xi}}
$$

and it becomes independent of $\bar{u}^{0}$ and $\bar{u}^{1}$. We seek the energy minimizer of (42) in the class of displacements

$$
V_{0}=\left\{\left(\tilde{u}_{ \pm}\right) \in H\left(\Omega_{ \pm} ; R^{3}\right): \tilde{u}_{+}\left(\hat{\tilde{\xi}},\left(\xi_{30}+\frac{1}{2}\right)^{+}\right)=\tilde{u}_{-}\left(\hat{\tilde{\xi}},\left(\xi_{30}-\frac{1}{2}\right)^{-}\right), \hat{\tilde{\xi}} \in S, \tilde{u}_{ \pm}=0 \text { on } \Gamma_{u}\right\} .
$$

Using standard arguments, we obtain that stationary points of the energy (42) satisfy the following equilibrium equations:

$$
\begin{aligned}
& \operatorname{div}\left(\mathbf{a}_{ \pm}\left(e\left(\tilde{u}_{ \pm}^{0}\right)\right)+\tilde{f}=0 \text { in } \Omega_{ \pm},\right. \\
& \mathbf{a}_{ \pm}\left(e\left(\tilde{u}_{ \pm}^{0}\right)\right) n=\tilde{g} \text { on } \Gamma_{g}, \\
& \mathbf{a}_{ \pm}\left(e\left(\tilde{u}_{ \pm}^{0}\right)\right) n=0 \text { on } \partial \Omega_{ \pm} \backslash \Gamma_{g}, \\
& {\left[\tilde{\sigma}^{0} \hat{\mathbf{e}}_{3}\right]=0 \text { on } S .}
\end{aligned}
$$

From the mechanical viewpoint, relations (41) and (47) correspond to conditions of perfect interface for the rescaled interphase modeling.

\subsection{Stationary points of $\mathcal{E}^{1}$}

The relations (38), (44)-(47) allow to simplify $\mathcal{E}^{1}$ as follows:

$$
\mathcal{E}^{1}=\int_{B}\left(\frac{1}{2} \mathbf{B}^{33}\left(\bar{u}_{, 3}^{1}\right) \cdot\left(\bar{u}_{, 3}^{1}\right)+\frac{1}{2} \mathbf{B}^{\alpha 3}\left(\bar{v}_{\alpha}^{0}\right) \cdot\left(\bar{u}_{, 3}^{1}\right)\right) \bar{H}^{0} d V_{\bar{\xi}}
$$

Stationary points $\bar{u}^{1}$ of $\mathcal{E}^{1}$ in $H\left(B ; R^{3}\right)$ satisfy the condition

$$
\tilde{\sigma}^{0} \hat{\mathbf{e}}_{3}=\mathbf{B}^{33}\left(\bar{u}_{, 3}^{1}\right)+\mathbf{B}^{\alpha 3}\left(\bar{v}_{\alpha}^{0}\right)
$$

with $\tilde{\sigma}^{0} \hat{\mathbf{e}}_{3}$ the common value of the tractions on $S_{ \pm}$. We integrate relation (49) along $\bar{\xi}_{3}$ taking into account that the fields at the order zero are independent of the coordinate $\xi_{3}$, and we use the continuity of the rescaled displacement vector fields across $S_{ \pm}$, implying that $\bar{v}_{\alpha}^{0}=\tilde{v}_{\alpha}^{0}$ on $S$, to obtain

$$
\left[\tilde{u}^{1}\right]=\left(\mathbf{B}^{33}\right)^{-1}\left(\tilde{\sigma}^{0} \hat{\mathbf{e}}_{3}-\mathbf{B}^{\alpha 3}\left(\tilde{v}_{\alpha}^{0}\right)\right) \quad \text { on } S .
$$

\subsection{Stationary points of $\mathcal{E}^{2}$}

Using (38) and (44)-(47), we simplify $\mathcal{E}^{2}$ as follows:

$$
\mathcal{E}^{2}=\int_{\Omega_{ \pm}} \frac{1}{2} \mathbf{a}_{ \pm}\left(e\left(\tilde{u}_{ \pm}^{1}\right)\right) \cdot e\left(\tilde{u}_{ \pm}^{1}\right) \tilde{H}^{0} d V_{\tilde{\xi}}+\int_{B}\left(\mathbf{B}^{3 \alpha}\left(\bar{u}_{, 3}^{1}\right)+\mathbf{B}^{\beta \alpha}\left(\bar{v}_{\beta}^{0}\right)\right) \cdot\left(\bar{v}_{\alpha}^{1}\right) \bar{H}^{0} d V_{\bar{\xi}}
$$


Noting the relation

$$
\bar{v}_{\alpha}^{1}=\frac{\bar{u}_{, \alpha}^{1}}{\bar{h}_{\alpha}^{0}}+\bar{\eta}_{\alpha}^{0}\left(\bar{u}^{1}\right)-\frac{\bar{h}_{\alpha}^{1}}{\left(\bar{h}_{\alpha}^{0}\right)^{2}} \bar{u}_{, \alpha}^{0}+\bar{\eta}_{\alpha}^{0}\left(\bar{u}^{0}\right),
$$

we observe that the second integral in (51) is given by

$$
\int_{B}\left(\frac{1}{2} \mathbf{B}^{3 \alpha}\left(\bar{u}_{, 3}^{1}\right)+\mathbf{B}^{\beta \alpha}\left(\bar{v}_{\beta}^{0}\right)\right) \cdot\left(\frac{\bar{u}_{, \alpha}^{1}}{\bar{h}_{\alpha}^{0}}+\bar{\eta}_{\alpha}^{0}\left(\bar{u}^{1}\right)\right) \bar{H}^{0} d V_{\bar{\xi}} .
$$

up to (constant) terms in $\bar{u}^{0}$ and its first derivatives. In view of (49) and under suitable regularity assumptions, the field $\bar{u}^{1}$ admits the following representation

$$
\bar{u}^{1}\left(\hat{\bar{\xi}}, \bar{\xi}_{3}\right)=\left[\tilde{u}^{1}\right] \bar{\xi}_{3}+\tilde{w}\left(\tilde{u}^{1}\right)(\hat{\bar{\xi}})
$$

where $\left.\left.\tilde{w}(\mathfrak{f}):=(1 / 2)\left(\mathfrak{f}\left(\xi_{30}+(1 / 2)\right)^{+}\right)+\mathfrak{f}\left(\xi_{30}-(1 / 2)\right)^{-}\right)\right)$for a generic function $\mathfrak{f}: \Omega_{+} \cup \Omega_{-} \mapsto R^{3}$. Integrating (54) along $\bar{\xi}_{3}$ simplifies the term linear in $\bar{\xi}_{3}$ and gives

$$
\mathcal{E}^{\prime 2}=\int_{\Omega_{ \pm}} \frac{1}{2} \mathbf{a}_{ \pm}\left(e\left(\tilde{u}_{ \pm}^{1}\right)\right) \cdot e\left(\tilde{u}_{ \pm}^{1}\right) \tilde{H}^{0} d V_{\tilde{\xi}}+\int_{S}\left(\mathbf{B}^{3 \alpha}\left(\bar{u}_{, 3}^{1}\right)+\mathbf{B}^{\beta \alpha}\left(\bar{v}_{\beta}^{0}\right)\right) \cdot \frac{1}{\bar{h}_{\alpha}^{0}}\left(\tilde{w}\left(\tilde{u}^{1}\right)\right)_{, \alpha}+\bar{\eta}_{\alpha}^{0}\left(\tilde{w}\left(\tilde{u}^{1}\right)\right) \bar{H}^{0} d V_{\bar{\xi}}
$$

up to terms in $\bar{u}^{0}$, which are considered constant in the minimization procedure. To "complete" the gradient of $\tilde{w}\left(\tilde{u}^{1}\right)$, we add to the second integral the term $\left(\left(\tilde{\sigma}^{0} \hat{\mathbf{e}}_{3}\right) \cdot\left(\tilde{w}\left(\tilde{u}^{1}\right)\right)_{, 3}\right)$, which vanishes being $\tilde{w}\left(\tilde{u}^{1}\right)$ independent of $\bar{\xi}_{3}$. We obtain

$$
\mathcal{E}^{2}=\int_{\Omega_{ \pm}} \frac{1}{2} \mathbf{a}_{ \pm}\left(e\left(\tilde{u}_{ \pm}^{1}\right)\right) \cdot e\left(\tilde{u}_{ \pm}^{1}\right) \tilde{H}^{0} d V_{\tilde{\xi}}+\int_{S}\left(\mathbf{M} \cdot \nabla \tilde{w}\left(\tilde{u}^{1}\right)\right) \bar{H}^{0} d V_{\bar{\xi}}
$$

where

$$
\mathbf{M}:=\left(\mathbf{B}^{31}\left(\bar{u}_{, 3}^{1}\right)+\mathbf{B}^{\beta 1}\left(\bar{v}_{\beta}^{0}\right)\left|\mathbf{B}^{32}\left(\bar{u}_{, 3}^{1}\right)+\mathbf{B}^{\beta 2}\left(\bar{v}_{\beta}^{0}\right)\right| \tilde{\sigma}^{0} \hat{\mathbf{e}}_{3}\right) .
$$

Finally, using standard arguments, we find that stationary points of (56) satisfy the following equilibrium equations:

$$
\begin{aligned}
& \operatorname{div}\left(\mathbf{a}_{ \pm}\left(e\left(\tilde{u}_{ \pm}^{1}\right)\right)\right)=0 \text { in } \Omega_{ \pm}, \\
& \mathbf{a}_{ \pm}\left(e\left(\tilde{u}_{ \pm}^{1}\right)\right) n=0 \text { on } \partial \Omega_{ \pm} \backslash S_{ \pm}, \\
& {\left[\tilde{\sigma}^{1} \hat{\mathbf{e}}_{3}\right]=-\operatorname{div} \mathbf{M} \text { on } S,} \\
& \mathbf{M} n=0 \text { on } \partial S .
\end{aligned}
$$

Relations (50) and (60) are imperfect interface conditions modeling the mechanical behavior of the anisotropic interphase at the first order of the asymptotic expansion. As a final remark, we note that condition (61) implies that the asymptotic expansions (17) and (18) do not hold in a neighborhood of $\partial S$ (Lebon and Rizzoni, 2010, Section 3).

\section{Imperfect interface conditions arising from an isotropic and homogeneous interphase}

In this section the conditions (50) and (60) obtained in Section 3 are specialized for the case of an isotropic and homogeneous interphase with Lamé coefficients $\mu$ and $\lambda$. In view of the definition (15), the matrices $\mathbf{B}^{i j}$ are given by

$$
\begin{aligned}
& \mathbf{B}^{i i}=(2 \mu+\lambda) \quad \hat{\mathbf{e}}_{i} \otimes \hat{\mathbf{e}}_{i}+\mu \quad\left(\hat{\mathbf{e}}_{j} \otimes \hat{\mathbf{e}}_{j}+\hat{\mathbf{e}}_{k} \otimes \hat{\mathbf{e}}_{k}\right), \quad i \neq j \neq k \\
& \mathbf{B}^{i j}=\mu \quad \hat{\mathbf{e}}_{i} \otimes \hat{\mathbf{e}}_{j}+\lambda \quad \hat{\mathbf{e}}_{j} \otimes \hat{\mathbf{e}}_{i}, \quad i \neq j .
\end{aligned}
$$

Substituting these relations into (50) gives the following expressions for the jumps in the displacement components:

$$
\begin{aligned}
& {\left[\tilde{u}_{1}^{1}\right]=\frac{1}{\mu} \tilde{\sigma}_{13}^{0}-\frac{1}{\bar{h}_{1}^{0}} \tilde{u}_{3,1}^{0}+\frac{\bar{h}_{1,3}^{0}}{\bar{h}_{1}^{0}} \tilde{u}_{1}^{0},} \\
& {\left[\tilde{u}_{2}^{1}\right]=\frac{1}{\mu} \tilde{\sigma}_{23}^{0}-\frac{1}{\bar{h}_{2}^{0}} \tilde{u}_{3,2}^{0}+\frac{\bar{h}_{2,3}^{0}}{\bar{h}_{2}^{0}} \tilde{u}_{2}^{0},} \\
& {\left[\tilde{u}_{3}^{1}\right]=\frac{1}{(2 \mu+\lambda)} \tilde{\sigma}_{33}^{0}-\frac{\lambda}{(2 \mu+\lambda)}\left(\frac{1}{\bar{h}_{1}^{0}} \tilde{u}_{1,1}^{0}+\frac{1}{\bar{h}_{2}^{0}} \tilde{u}_{2,2}^{0}+\frac{\bar{h}_{1,2}^{0}}{\bar{h}_{1}^{0} \bar{h}_{2}^{0}} \tilde{u}_{2}^{0}+\frac{\bar{h}_{2,1}^{0}}{\bar{h}_{1}^{0} \bar{h}_{2}^{0}} \tilde{u}_{1}^{0}+\left(\frac{\bar{h}_{1,3}^{0}}{\bar{h}_{1}^{0}}+\frac{\bar{h}_{2,3}^{0}}{\bar{h}_{2}^{0}}\right) \tilde{u}_{3}^{0}\right) .}
\end{aligned}
$$


Substituting (62) and (63) into (60) and using the expression of the divergence of a tensor given in Bolton (1993), we have the following relations for the jumps of the stress components at order one:

$$
\begin{aligned}
& {\left[\tilde{\sigma}_{13}^{1}\right]=-\left(\frac{1}{\bar{h}_{1}^{0}} M_{11,1}+\frac{1}{\bar{h}_{2}^{0}} M_{12,2}+\frac{\bar{h}_{1,2}^{0}}{\bar{h}_{1}^{0} \bar{h}_{2}^{0}}\left(M_{12}+M_{21}\right)+\frac{\bar{h}_{2,1}^{0}}{\bar{h}_{1}^{0} \bar{h}_{2}^{0}}\left(M_{11}-M_{22}\right)+\frac{\bar{h}_{1,3}^{0}}{\bar{h}_{1}^{0}} M_{31}+\left(\frac{\bar{h}_{1,3}^{0}}{\bar{h}_{1}^{0}}+\frac{\bar{h}_{2,3}^{0}}{\bar{h}_{2}^{0}}\right) M_{13}\right),} \\
& {\left[\tilde{\sigma}_{23}^{1}\right]=-\left(\frac{1}{\bar{h}_{1}^{0}} M_{21,1}+\frac{1}{\bar{h}_{2}^{0}} M_{22,2}+\frac{\bar{h}_{1,2}^{0}}{\bar{h}_{1}^{0} \bar{h}_{2}^{0}}\left(M_{22}-M_{11}\right)+\frac{\bar{h}_{2,1}^{0}}{\bar{h}_{1}^{0} \bar{h}_{2}^{0}}\left(M_{12}+M_{21}\right)+\frac{\bar{h}_{2,3}^{0}}{\bar{h}_{2}^{0}} M_{32}+\left(\frac{\bar{h}_{1,3}^{0}}{\bar{h}_{1}^{0}}+\frac{\bar{h}_{2,3}^{0}}{\bar{h}_{2}^{0}}\right) M_{23}\right),} \\
& {\left[\tilde{\sigma}_{33}^{1}\right]=-\left(\frac{1}{\bar{h}_{1}^{0}} M_{31,1}+\frac{1}{\bar{h}_{2}^{0}} M_{32,2}+\frac{\bar{h}_{1,2}^{0}}{\bar{h}_{1}^{0} \bar{h}_{2}^{0}} M_{32}+\frac{\bar{h}_{2,1}^{0}}{\bar{h}_{1}^{0} \bar{h}_{2}^{0}} M_{31}+\left(\frac{\bar{h}_{1,3}^{0}}{\bar{h}_{1}^{0}}+\frac{\bar{h}_{2,3}^{0}}{\bar{h}_{2}^{0}}\right) M_{33}-\frac{\bar{h}_{1,3}^{0}}{\bar{h}_{1}^{0}} M_{11}-\frac{\bar{h}_{2,3}^{0}}{\bar{h}_{2}^{0}} M_{22}\right),}
\end{aligned}
$$

where

$$
\begin{aligned}
& M_{11}=\frac{\lambda}{(2 \mu+\lambda)} \tilde{\sigma}_{33}^{0}+\frac{4 \mu(\mu+\lambda)}{(2 \mu+\lambda)}\left(\frac{1}{\bar{h}_{1}^{0}} \tilde{u}_{1,1}^{0}+\frac{\bar{h}_{1,2}^{0}}{\bar{h}_{1}^{0} \bar{h}_{2}^{0}} \tilde{u}_{2}^{0}+\frac{\bar{h}_{1,3}^{0}}{\bar{h}_{1}^{0}} \tilde{u}_{3}^{0}\right)+\frac{2 \mu \lambda}{(2 \mu+\lambda)}\left(\frac{1}{\bar{h}_{2}^{0}} \tilde{u}_{2,2}^{0}+\frac{\bar{h}_{2,1}^{0}}{\bar{h}_{1}^{0} \bar{h}_{1}^{0}} \tilde{u}_{2}^{0}+\frac{\bar{h}_{2,3}^{0}}{\bar{h}_{2}^{0}} \tilde{u}_{3}^{0}\right) \\
& M_{22}=\frac{\lambda}{(2 \mu+\lambda)} \tilde{\sigma}_{33}^{0}+\frac{2 \mu \lambda}{(2 \mu+\lambda)}\left(\frac{1}{\bar{h}_{1}^{0}} \tilde{u}_{1,1}^{0}+\frac{\bar{h}_{1,2}^{0}}{\bar{h}_{1}^{0} \bar{h}_{2}^{0}} \tilde{u}_{2}^{0}+\frac{\bar{h}_{1,3}^{0}}{\bar{h}_{1}^{0}} \tilde{u}_{3}^{0}\right)+\frac{4 \mu(\mu+\lambda)}{(2 \mu+\lambda)}\left(\frac{1}{\bar{h}_{2}^{0}} \tilde{u}_{2,2}^{0}+\frac{\bar{h}_{2,1}^{0}}{\bar{h}_{1}^{0} \bar{h}_{1}} \tilde{u}_{2}^{0}+\frac{\bar{h}_{2,3}^{0}}{\bar{h}_{2}^{0}} \tilde{u}_{3}^{0}\right) \\
& M_{12}=M_{21}=\mu\left(\frac{1}{\bar{h}_{2}^{0}} \tilde{u}_{1,2}^{0}+\frac{1}{\bar{h}_{1}^{0}} \tilde{u}_{2,1}^{0}-\frac{\bar{h}_{1,2}^{0}}{\bar{h}_{1}^{0} \bar{h}_{2}^{0}} \tilde{u}_{1}^{0}-\frac{\bar{h}_{2,1}^{0}}{\bar{h}_{1}^{0} \bar{h}_{2}^{0}} \tilde{u}_{1}^{0}\right) \\
& M_{i 3}=M_{3 i}=\tilde{\sigma}_{i 3}^{0}, \quad i=1,2,3 .
\end{aligned}
$$

\section{Implementation of the imperfect interface conditions}

In this section we show how the conditions (41), (47), (50) and (60) can be used to obtain imperfect interface conditions for the original (unrescaled) problem. Consider a minimizer, $u^{\varepsilon}$, of the energy (6), and a smooth extension of $u^{\varepsilon}$ to $\Omega_{ \pm}^{0}, \Gamma_{g}^{0}$ and $\Gamma_{u}^{0}$, which are the domains obtained by taking the geometric limit of $\Omega_{ \pm}^{\varepsilon}, \Gamma_{g}^{\varepsilon}$ and $\Gamma_{u}^{\varepsilon}$ as $\varepsilon \rightarrow 0^{+}$(Fig. 1c). Assume also the existence of an expansion in powers of $\varepsilon$ also for $u^{\varepsilon}$ :

$$
u^{\varepsilon}=u^{0}+\varepsilon u^{1}+\varepsilon^{2} u^{2}+o\left(\varepsilon^{2}\right)
$$

In view of the change of variable ((12) and (13)), we have

$$
u^{\varepsilon}\left(\hat{\xi},\left(\xi_{30} \pm \frac{\varepsilon}{2}\right)^{ \pm}\right)=\tilde{u}_{ \pm}^{\varepsilon}\left(\hat{\tilde{\xi}},\left(\xi_{30} \pm \frac{1}{2}\right)^{ \pm}\right), \quad \hat{\xi}, \hat{\tilde{\xi}} \in S .
$$

Substituting the expansions (17) and (74) into (75), we obtain

$$
u^{0}\left(\hat{\xi},\left(\xi_{30} \pm \frac{\varepsilon}{2}\right)^{ \pm}\right)+\varepsilon u^{1}\left(\hat{\xi},\left(\xi_{30} \pm \frac{\varepsilon}{2}\right)^{ \pm}\right)+o\left(\varepsilon^{2}\right)=\tilde{u}_{ \pm}^{0}\left(\hat{\tilde{\xi}},\left(\xi_{30} \pm \frac{1}{2}\right)^{ \pm}\right)+\varepsilon \tilde{u}_{ \pm}^{1}\left(\hat{\widetilde{\xi}},\left(\xi_{30} \pm \frac{1}{2}\right)^{ \pm}\right)+o\left(\varepsilon^{2}\right)
$$

Now we assume that the terms on the left-hand side can be expanded in powers of $\varepsilon$

$$
u^{0}\left(\hat{\xi}, \xi_{30}^{ \pm}\right) \pm \varepsilon\left(\frac{1}{2} u_{, 3}^{0}\left(\hat{\xi}, \xi_{30}^{ \pm}\right)+u^{1}\left(\hat{\xi}, \xi_{30}^{ \pm}\right)\right)+o\left(\varepsilon^{2}\right)=\tilde{u}_{ \pm}^{0}\left(\hat{\tilde{\xi}},\left(\xi_{30} \pm \frac{1}{2}\right)^{ \pm}\right)+\varepsilon \tilde{u}_{ \pm}^{1}\left(\hat{\tilde{\xi}},\left(\xi_{30} \pm \frac{1}{2}\right)^{ \pm}\right)+o\left(\varepsilon^{2}\right)
$$

and we identify the terms in $\varepsilon$ to get

$$
\begin{aligned}
& u^{0}\left(\hat{\xi}, \xi_{30}^{ \pm}\right)=\tilde{u}_{ \pm}^{0}\left(\hat{\tilde{\xi}},\left(\xi_{30} \pm \frac{1}{2}\right)^{ \pm}\right) \\
& \pm \frac{1}{2} u_{, 3}^{0}\left(\hat{\xi}, \xi_{30}^{ \pm}\right)+u^{1}\left(\hat{\xi}, \xi_{30}^{ \pm}\right)=\tilde{u}_{ \pm}^{1}\left(\hat{\tilde{\xi}},\left(\xi_{30} \pm \frac{1}{2}\right)^{ \pm}\right) .
\end{aligned}
$$

Therefore, we have

$$
\begin{aligned}
& {\left[\left[u^{0}\right]\right]=\left[\tilde{u}^{0}\right]} \\
& {\left[\left[u^{1}\right]\right]=\left[\tilde{u}^{1}\right]-w\left(u_{, 3}^{0}\right)}
\end{aligned}
$$


where $[[\mathfrak{f}]](\hat{\xi}):=\mathfrak{f}\left(\hat{\xi}, \xi_{30}^{+}\right)-\mathfrak{f}\left(\hat{\xi}, \xi_{30}^{-}\right)$is taken to denote the jump across $S$ of a generic function $\mathfrak{f}: \Omega_{+}^{0} \cup \Omega_{-}^{0} \mapsto R^{3}$ and $w(\mathfrak{f})(\hat{\xi}):=$ $(1 / 2)\left(\mathfrak{f}\left(\hat{\xi}, \xi_{30}^{+}\right)+\mathfrak{f}\left(\hat{\xi}, \xi_{30}^{-}\right)\right)$. Similar relations can be obtained for the tractions:

$$
\begin{aligned}
& \sigma_{ \pm}^{0}\left(\hat{\xi}, \xi_{30}^{ \pm}\right) \hat{\mathbf{e}}_{3}=\tilde{\sigma}_{ \pm}^{0}\left(\hat{\tilde{\xi}},\left(\xi_{30} \pm \frac{1}{2}\right)^{ \pm}\right) \hat{\mathbf{e}}_{3}, \\
& \pm \frac{1}{2} \sigma_{ \pm, 3}^{0}\left(\hat{\xi}, \xi_{30}^{ \pm}\right) \hat{\mathbf{e}}_{3}+\sigma_{ \pm}^{1}\left(\hat{\xi}, \xi_{30}^{ \pm}\right) \hat{\mathbf{e}}_{3}=\tilde{\sigma}_{ \pm}^{1}\left(\hat{\tilde{\xi}},\left(\xi_{30} \pm \frac{1}{2}\right)^{ \pm}\right) \hat{\mathbf{e}}_{3}, \\
& {\left[\left[\sigma^{0} \hat{\mathbf{e}}_{3}\right]\right]=\left[\tilde{\sigma}^{0} \hat{\mathbf{e}}_{3}\right],} \\
& {\left[\left[\sigma^{1} \hat{\mathbf{e}}_{3}\right]\right]=\left[\tilde{\sigma}^{1} \hat{\mathbf{e}}_{3}\right]-w\left(\sigma_{, 3}^{0} \hat{\mathbf{e}}_{3}\right) .}
\end{aligned}
$$

Substituting the conditions (41), (47), (50) and (60) into (80), (81), (84), (85) and using (78) and (82) we obtain the following interface conditions for the original limit problem:

$$
\begin{aligned}
& {\left[\left[u^{0}\right]\right]=0} \\
& {\left[\left[u^{1}\right]\right]=\left(\mathbf{B}^{33}\right)^{-1}\left(\sigma^{0} \hat{\mathbf{e}}_{3}-\mathbf{B}^{\alpha 3}\left(v_{\alpha}^{0}\right)\right)-w\left(u_{, 3}^{0}\right),} \\
& {\left[\left[\sigma^{0} \hat{\mathbf{e}}_{3}\right]\right]=0} \\
& {\left[\left[\sigma^{1} \hat{\mathbf{e}}_{3}\right]\right]=-\operatorname{div} \mathbf{M}-w\left(\sigma_{, 3}^{0} \hat{\mathbf{e}}_{3}\right),}
\end{aligned}
$$

with $\sigma^{0} \hat{\mathbf{e}}_{3}$ the common value of the tractions on both sides of S. Using again the change of variables (12) and (13) in (44)-(46), (58) and (59), we obtain the following two equilibrium problems posed only on the limit configurations of the adherents and each in which the interphase is treated as a surface:

$$
\begin{aligned}
& \left(\mathcal{P}_{0}\right) \begin{cases}\operatorname{div}\left(\mathbf{a}_{ \pm}\left(e\left(u^{0}\right)\right)+f=0\right. & \text { in } \Omega_{ \pm}^{0}, \\
\mathbf{a}_{ \pm}\left(e\left(u^{0}\right)\right) n=g & \text { on } \Gamma_{g}^{0} \\
u^{0}=0 & \text { on } \Gamma_{u}^{0} \\
{\left[\left[u^{0}\right]\right]=0} & \text { on } S, \\
{\left[\left[\sigma^{0} \hat{\mathbf{e}}_{3}\right]\right]=0} & \text { on } S,\end{cases} \\
& \left(\mathcal{P}_{1}\right) \begin{cases}\operatorname{div}\left(\mathbf{a}_{ \pm}\left(e\left(u^{1}\right)\right)=0\right. & \text { in } \Omega_{ \pm}^{0}, \\
\mathbf{a}_{ \pm}\left(e\left(u^{1}\right)\right) n=0 & \text { on } \Gamma_{g}^{0} \\
u^{1}=0 & \text { on } \Gamma_{u}^{0} \\
{\left[\left[u^{1}\right]\right]=\left(\mathbf{B}^{33}\right)^{-1}\left(\sigma^{0} \hat{\mathbf{e}}_{3}-\mathbf{B}^{\alpha 3}\left(v_{\alpha}^{0}\right)\right)-w\left(u_{, 3}^{0}\right)} & \text { on } S, \\
{\left[\left[\sigma^{1} \hat{\mathbf{e}}_{3}\right]\right]=-\operatorname{div} \mathbf{M}-w\left(\sigma_{, 3}^{0} \hat{\mathbf{e}}_{3}\right)} & \text { on } S .\end{cases}
\end{aligned}
$$

\section{Composite spheres assemblage}

To illustrate the implementation of the imperfect interface conditions developed so far, we consider the boundary value problem of a composite spheres assemblage subject to uniform radial displacement of magnitude $\delta$ on the outer boundary. The problem will be solved in two ways: first, exactly, as a three-phase elastic problem where the phases are a sphere of material "+", a concentric interphase, and an inner spherical inclusion of material "-", and second, as a two-phase problem, where the phases are the two concentric spheres of materials "+" and "-" with in between imperfect interface conditions.

We take $R_{e}, R_{0}+\varepsilon / 2$, to denote the exterior and the core radii of the outer sphere, and $R_{0}-\varepsilon / 2$ to denote the exterior radius of the inclusion. Let $\kappa_{+}, \mu_{+}$denote the elastic bulk and shear moduli of the outer spherical shell, $\kappa, \mu$ the elastic bulk and shear moduli of the interphase, and $\kappa_{-}, \mu_{-}$the elastic bulk and shear moduli of the inclusion.

The general three-dimensional equations of homogeneous, isotropic linearized elasticity have the following general solution in terms of spherical coordinates $r, \theta, \phi$ (Lakes and Drugan, 2002):

$$
\begin{aligned}
& u_{r}=\alpha r+\frac{\beta}{r^{2}}, \\
& \epsilon_{r r}=\alpha-2 \frac{\beta}{r^{3}}, \quad \epsilon_{\theta \theta}=\epsilon_{\phi \phi}=\alpha+\frac{\beta}{r^{3}},
\end{aligned}
$$




$$
\sigma_{r r}=3 \kappa \alpha-4 \mu \frac{\beta}{r^{3}}, \quad \sigma_{\theta \theta}=\sigma_{\phi \phi}=3 \kappa \alpha+2 \mu \frac{\beta}{r^{3}},
$$

where $\alpha$ and $\beta$ are initially undetermined constants. These solutions apply inside the three phases, with appropriate values of the elastic moduli and with different values of the constants $\alpha, \beta$.

Let $\alpha_{ \pm}^{\varepsilon}, \beta_{ \pm}^{\varepsilon}, \alpha^{\varepsilon}, \beta^{\varepsilon}$ denote the six constants of the three-phase elastic problem. The condition $\beta_{-}^{\varepsilon}=0$ is necessary to avoid singularities at $r=0$. The remaining five constants are determined by imposing the boundary condition $u_{r}\left(R_{e}\right)=\delta$ and the continuity of the radial displacement $u_{r}$ and of the radial stress $\sigma_{r r}$ at $r=R_{0} \pm \frac{\varepsilon}{2}$. We obtain the following conditions:

$$
\begin{aligned}
& \alpha_{-}^{\varepsilon}\left(R_{0}-\frac{\varepsilon}{2}\right)=\alpha^{\varepsilon}\left(R_{0}-\frac{\varepsilon}{2}\right)+\frac{\beta^{\varepsilon}}{\left(R_{0}-\varepsilon / 2\right)^{2}}, \\
& 3 \kappa_{-} \alpha_{-}^{\varepsilon}=3 \kappa \alpha^{\varepsilon}-4 \mu \frac{\beta^{\varepsilon}}{\left(R_{0}-\varepsilon / 2\right)^{3}}, \\
& \alpha^{\varepsilon}\left(R_{0}-\frac{\varepsilon}{2}\right)+\frac{\beta^{\varepsilon}}{\left(R_{0}+\varepsilon / 2\right)^{2}}=\alpha_{+}^{\varepsilon}\left(R_{0}-\frac{\varepsilon}{2}\right)+\frac{\beta_{+}^{\varepsilon}}{\left(R_{0}+\varepsilon / 2\right)^{2}}, \\
& 3 \kappa \alpha^{\varepsilon}-4 \mu \frac{\beta^{\varepsilon}}{\left(R_{0}+\varepsilon / 2\right)^{3}}=3 \kappa_{+} \alpha_{+}^{\varepsilon}-4 \mu \frac{\beta_{+}^{\varepsilon}}{\left(R_{0}+\varepsilon / 2\right)^{3}}, \\
& \alpha_{+}^{\varepsilon} R_{e}+\frac{\beta_{+}^{\varepsilon}}{R_{e}^{2}}=\delta .
\end{aligned}
$$

Next, the perfect interface conditions calculated at order zero (cf. problem $\left(\mathcal{P}_{0}\right)$ ) are applied to the same problem. Let $\alpha_{ \pm}^{0}, \beta_{ \pm}^{0}$ denote the four constants of this two-phase elastic problem. Again, the condition $\beta_{-}^{0}=0$ is necessary to avoid singularities at $r=0$. The remaining three constants are determined by imposing the boundary condition and the continuity of the radial displacement and of the radial stress at $r=R_{0}$. We obtain the following conditions:

$$
\begin{aligned}
& \alpha_{-}^{0} R_{0}=\alpha_{+}^{0} R_{0}+\frac{\beta_{+}^{0}}{R_{0}^{2}}, \\
& 3 \kappa_{-} \alpha_{-}^{0}=3 \kappa_{+} \alpha_{+}^{0}-4 \mu_{+} \frac{\beta_{+}^{0}}{R_{0}^{3}}, \\
& \alpha_{+}^{0} R_{e}+\frac{\beta_{+}^{0}}{R_{e}^{2}}=\delta .
\end{aligned}
$$

Lastly, we solve two-phase elastic problem with the imperfect interface conditions calculated at order one (cf. problem $\left.\left(\mathcal{P}_{1}\right)\right)$. In the $\operatorname{spherical}$ coordinate system, we have

$$
h_{1}:=h_{\phi}=r \sin \theta, \quad h_{2}:=h_{\theta}=r, \quad h_{3}:=h_{r}=1 .
$$

Using (64)-(73) and (103), the imperfect interface conditions (91) $)_{4}$ and $(91)_{5}$ assume the form

$$
\begin{aligned}
& {\left[\left[u_{r}^{1}\right]\right]=\frac{3}{(3 \kappa+4 \mu)} \sigma_{r r}^{0}\left(R_{0}\right)-2 \frac{(3 \kappa-2 \mu)}{(3 \kappa+4 \mu)} \frac{u_{r}^{0}\left(R_{0}\right)}{R_{0}}-\frac{1}{2}\left(u_{r, r}^{0}\left(R_{0}^{+}\right)+u_{r, r}^{0}\left(R_{0}^{-}\right)\right),} \\
& {\left[\left[\sigma_{r r}^{1}\right]\right]=-\frac{12 \mu}{(3 \kappa+4 \mu)} \frac{\sigma_{r r}^{0}\left(R_{0}\right)}{R_{0}}+\frac{36 \kappa \mu}{(3 \kappa+4 \mu)} \frac{u_{r}^{0}\left(R_{0}\right)}{R_{0}^{2}}-\frac{1}{2}\left(\sigma_{r r, r}^{0}\left(R_{0}^{+}\right)+\sigma_{r r, r}^{0}\left(R_{0}^{-}\right)\right) .}
\end{aligned}
$$

Let $\alpha_{ \pm}^{1}, \beta_{ \pm}^{1}$ denote the four constants of the two-phase elastic problem with the imperfect interface conditions. We still find that the condition $\beta_{-}^{1}=0$ avoids singularities at $r=0$. The remaining three constants are determined by imposing (104) and (105), and the boundary condition at $r=R_{e}$ with $\delta=0$. We obtain the following conditions:

$$
\begin{aligned}
& \alpha_{+}^{1} R_{0}+\frac{\beta_{+}^{1}}{R_{0}^{2}}-\alpha_{-}^{1} R_{0}=\frac{9 \kappa_{-} \alpha_{-}^{0}}{(3 \kappa+4 \mu)}-2 \frac{(3 \kappa-2 \mu)}{(3 \kappa+4 \mu)} \alpha_{-}^{0}-\frac{1}{2}\left(\alpha_{+}^{0}-\frac{2 \beta_{+}^{0}}{R_{0}^{3}}+\alpha_{-}^{0}\right), \\
& 3 \kappa_{+} \alpha_{+}^{1}-4 \mu_{+} \frac{\beta_{+}^{1}}{R_{0}^{3}}-3 \kappa_{-} \alpha_{-}^{1}=\frac{36 \mu\left(\kappa-\kappa_{-}\right)}{(3 \kappa+4 \mu)} \frac{\alpha_{-}^{0}}{R_{0}}-\frac{6 \mu_{+} \beta_{+}^{0}}{R_{0}^{4}}, \\
& \alpha_{+}^{1} R_{e}+\frac{\beta_{+}^{1}}{R_{e}^{2}}=0 .
\end{aligned}
$$

Comparisons between the radial and circumferential stresses as functions of $\xi=\log _{10} \kappa / \kappa_{+}$on the external surface $r=R_{e}$ calculated from the exact three-phase solution, the perfect and the imperfect interface approximations are given in Figs. 2 and 3 for the two 


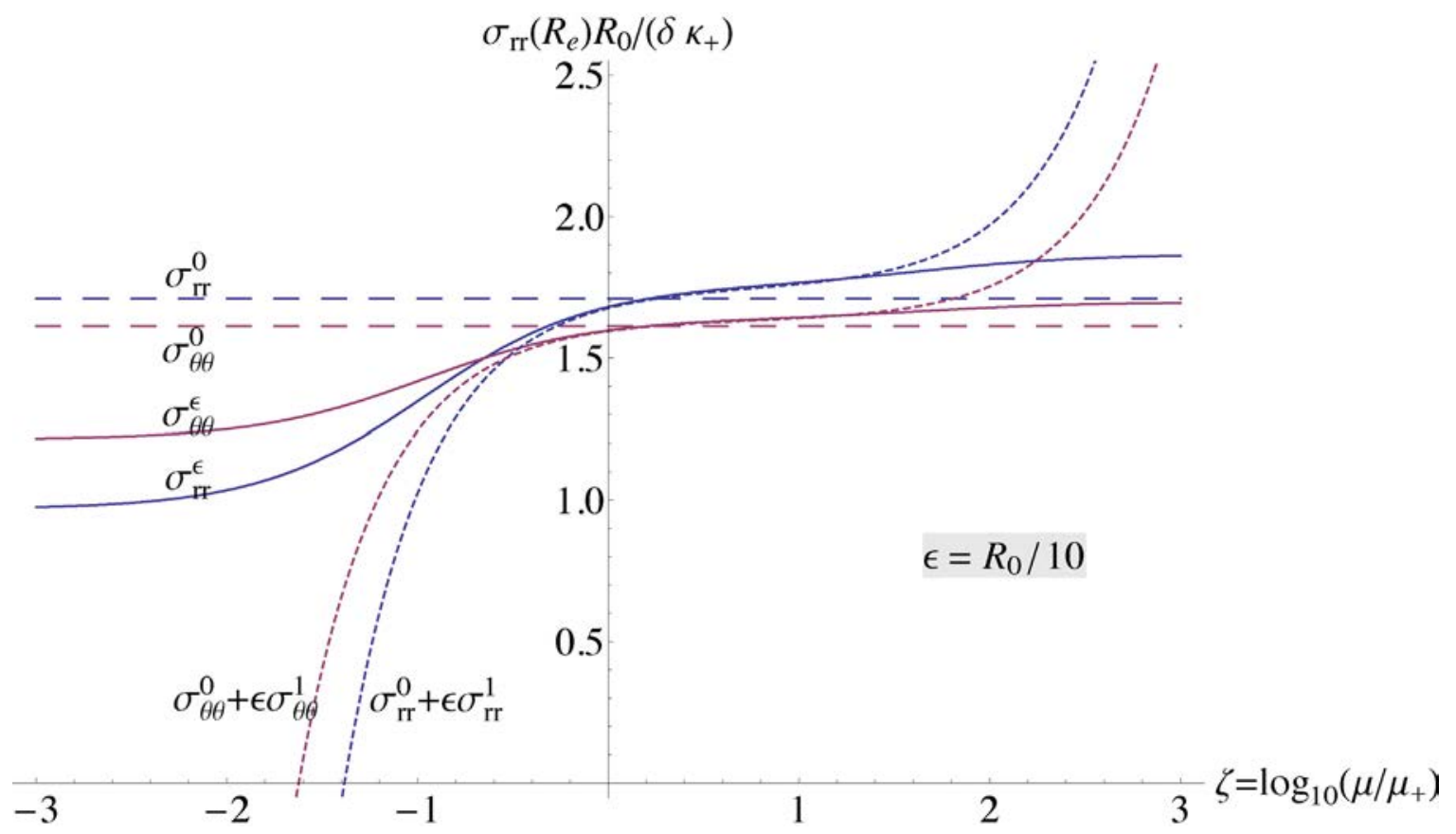

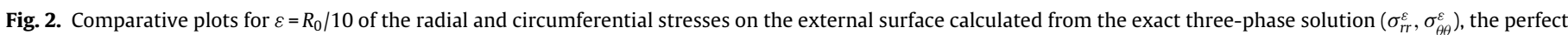
$\left(\sigma_{r r}^{0}, \sigma_{\theta \theta}^{0}\right)$ and the imperfect $\left(\sigma_{r r}^{0}+\varepsilon \sigma_{r r}^{1}, \sigma_{\theta \theta}^{0}+\varepsilon \sigma_{\theta \theta}^{1}\right)$ interface approximations.

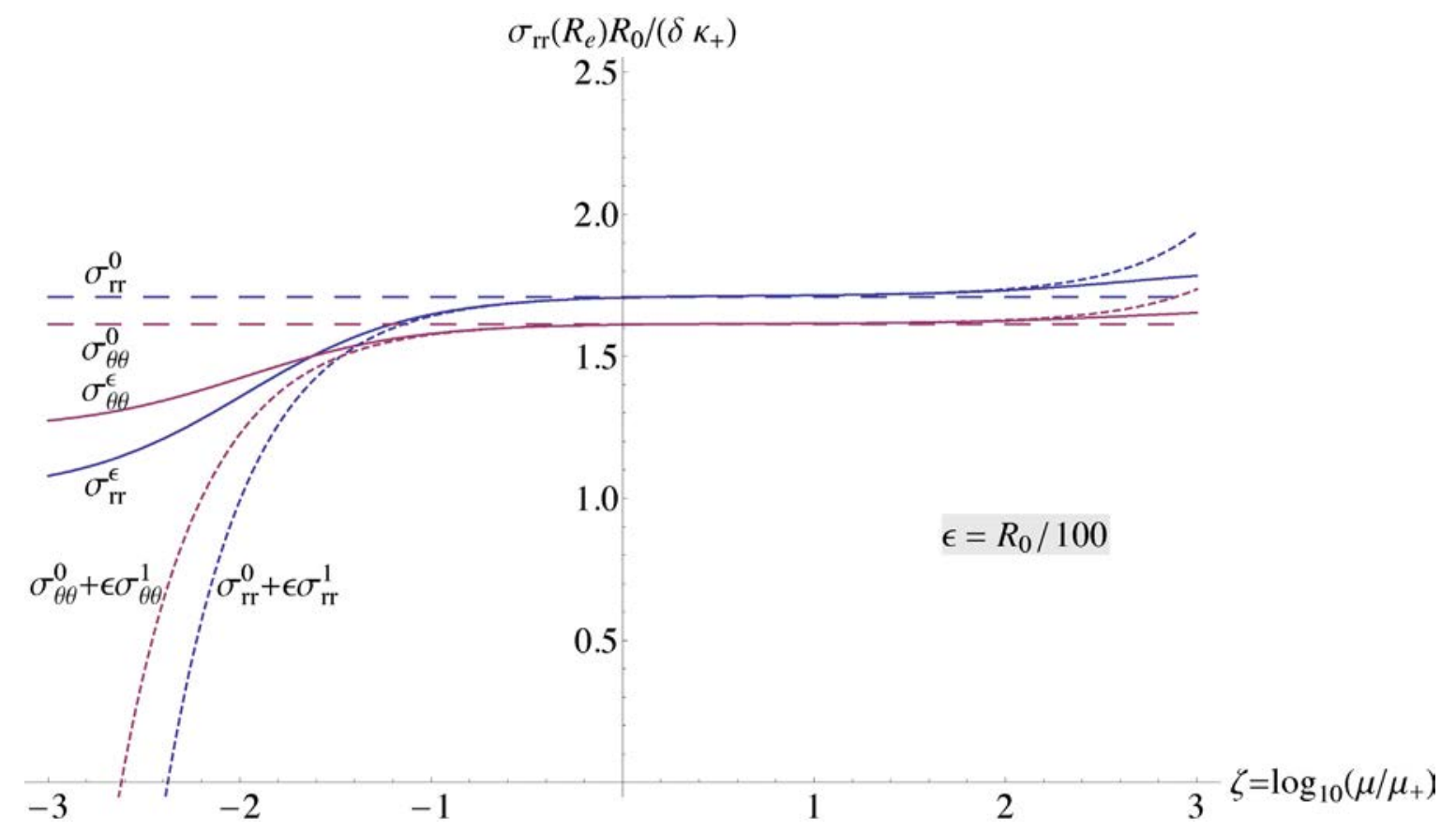

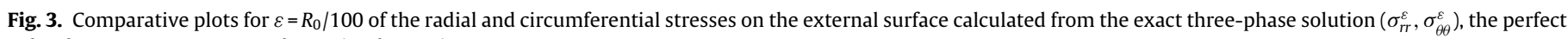
$\left(\sigma_{r r}^{0}, \sigma_{\theta \theta}^{0}\right)$ and the imperfect $\left(\sigma_{r r}^{0}+\varepsilon \sigma_{r r}^{1}, \sigma_{\theta \theta}^{0}+\varepsilon \sigma_{\theta \theta}^{1}\right)$ interface approximations.

cases $\varepsilon=0.1 R_{0}$ and $\varepsilon=0.01 R_{0}$. For drawing the plots, the following values for the material and geometrical constants were also assumed:

$$
\begin{aligned}
& \mu_{-}=10 \quad \mu_{+}, \\
& \nu_{-}=0.20, \quad v_{+}=0.35, \quad v=0.3, \\
& R_{e}=2 R_{0},
\end{aligned}
$$


where $v_{ \pm}$are the Poisson coefficients of the materials " \pm " and $v$ is the Poisson coefficient of the interphase material. It should be noted that, for the values of $\varepsilon$ considered in Figs. 2 and 3, the imperfect interface model is more accurate than the perfect interface model for $\mu / \mu_{+}$ranging from $10^{-1}$ to $10^{1}$.

\section{Conclusions}

In this study, a model of a curved thin elastic interphase is formulated which is characterized by two-level interface conditions. At the first level, the interphase is replaced by a model of perfect interface, for which the traction and the displacement vector fields are continuous (cf. conditions $(90)_{4}$ and $\left.(90)_{5}\right)$. At the second level, the jumps in the displacements and in the stress vector along the interface are related to the interphase material properties and to the displacement and stress fields obtained at the first level (cf. conditions (91) 4 , $\left.(91)_{5}\right)_{\text {. The }}$ interface conditions are obtained in a system of parallel orthogonal curvilinear coordinates and are given in terms of the displacements and tractions belonging to the adherents and evaluated at both sides of the interphase. The main advantage of the interface model is that it makes possible to solve for the fields in the adherents without having to solve for the fields in the interphase. To illustrate its implementation, a spherical composite assemblage is studied and comparisons between the radial and circumferential stresses computed from the exact three-phase solution, the perfect and the imperfect interface approximations are given. A motivation for the study of interface models is the complexity of the finite element modeling due to the thinness of the thin layer, whose presence causes an ill-conditionment of the tangent matrices and affects the precision of the computations. The computational cost may be amplified by the differences between the material properties of the interphase and those of the adherents. The use of the imperfect interface model brings the intrinsic advantage of reducing the numerical analysis to a two-phase equilibrium problem. The imperfect interface laws obtained in this paper could also been advantageously used to evaluated the effect of coating interphases on the effective properties of composites. Implementation of the two-level model in a finite element software and a study the singularities at the edges of the interface will be addressed in a future work.

\section{Acknowledgments}

RR thanks the financial support of the Italian Ministry of Education, University and Research (MIUR) through PRIN project "Multi-scale modeling of materials and structures" (code 2010BFXRHS).

\section{References}

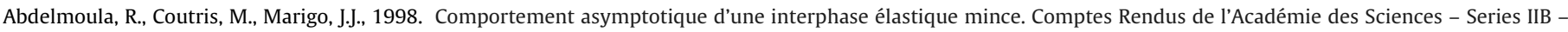
Mechanics 326, 237-242.

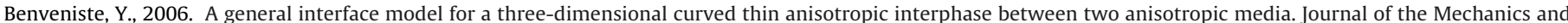
Physics of Solids 54, 708-734.

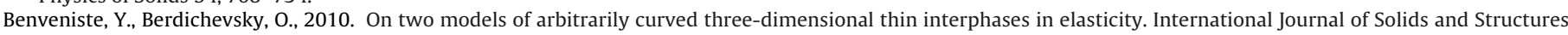
47, 1899-1915.

Benveniste, Y., Miloh, T., 2001. Imperfect soft and stiff interfaces in two-dimensional elasticity. Mechanics of Materials 33, $309-323$.

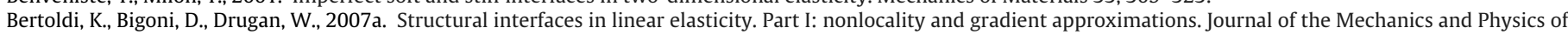
Solids 55, 1-34.

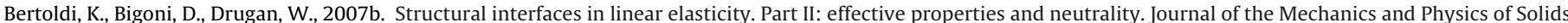
55, 35-63.

Bigoni, D., Movchan, A., 2002. Statics and dynamics of structural interfaces in elasticity. International Journal of Solids and Structures $39,4843-4865$.

Bolton, E.W., 1993. A simple notation for differential vector expressions in orthogonal curvilinear coordinates. Geophysical Journal International, 115.

Caillerie, D., 1980. The effect of a thin inclusion of high rigidity in an elastic body. Mathematical Methods in the Applied Sciences 2, 251-270.

Ciarlet, P.G., 1988. Mathematical Elasticity. Volume I: Three-Dimensional Elasticity. North-Holland.

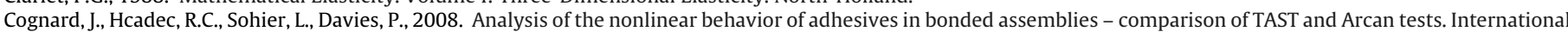
Journal of Adhesion and Adhesives 28, 393-404.

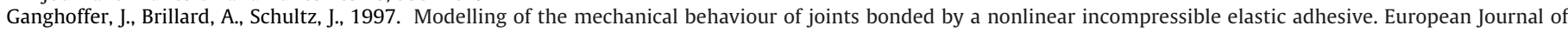
Mechanics - A/Solids 16, 255-276.

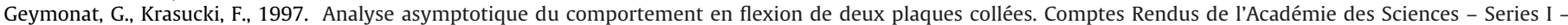
Mathematics 325, 307-314.

Geymonat, G., Krasucki, F., Lenci, S., 1999. Mathematical analysis of a bonded joint with a soft thin adhesive. Mathematics and Mechanics of Solids 4, $201-225$.

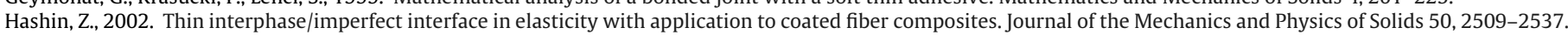

Klarbring, A., 1991. Derivation of the adhesively bonded joints by the asymptotic expansion method. International Journal of Engineering Science $29,493-512$.

Klarbring, A., Movchan, A., 1998. Asymptotic modelling of adhesive joints. Mechanics of Materials 28, 137-145.

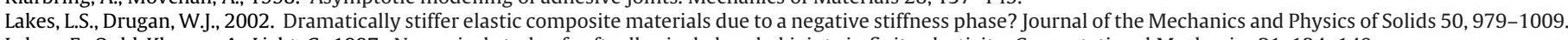

Lebon, F., Ould-Khaoua, A., Licht, C., 1997. Numerical study of soft adhesively bonded joints in finite elasticity. Computational Mechanics 21, $134-140$.

Lebon, F., Rizzoni, R, 2008. Asymptotic study of soft thin layer: the non convex case. Mechanics of Advanced Materials and Structures 15, 12-20.

Lebon, F., Rizzoni, R., 2010. Asymptotic analysis of a thin interface: the case involving similar rigidity. International Journal of Engineering Sciences $48,473-486$.

Lebon, F., Rizzoni, R., 2011. Asymptotic behavior of a hard thin linear elastic interphase: an energy approach. International Journal of Solids and Structures $48,441-449$

Lebon, F., Rizzoni, R., Ronel, S., 2004. Analysis of non-linear soft thin interfaces. Computers \& Structures 82, 1929-1938.

Lebon, F., Ronel, S., 2007. First order numerical analysis of linear thin layers. ASME Journal of Applied Mechanics 74, 824-828.

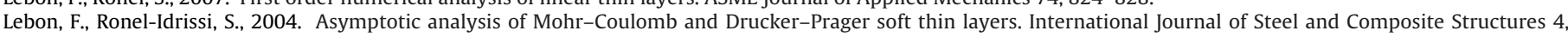
$133-148$.

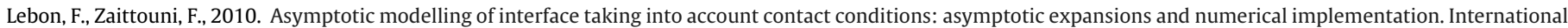
Journal of Engineering Sciences 48, 111-127.

Lenci, S., 2000. A limit model in the analysis of steel-concrete beams. Mathematical Models and Methods in Applied Sciences 10, $1233-1250$.

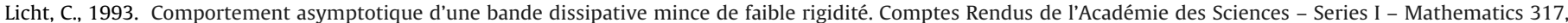
429-433.

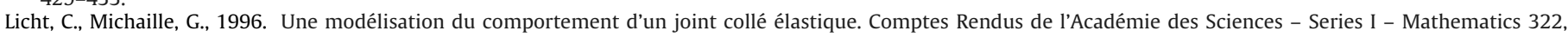
295-300.

Licht, C., Michaille, G., 1997. A modeling of elastic adhesive bonded joints. Advances in Mathematical Sciences and Applications 7, 711-740.

Monchiet, V., Bonnet, G., 2010. Interfacial models in viscoplastic composites materials. International Journal of Engineering Science 48, 1762-1768.

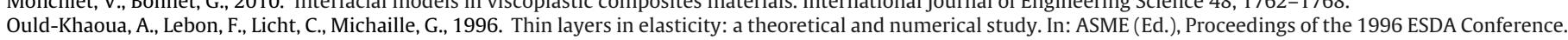
pp. 171-178.

Pelissou, C., Lebon, F., 2009. Asymptotic modeling of quasi-brittle interfaces. Computers \& Structures 87, 1216-1223.

Rekik, A., Lebon, F., 2012. Homogenization methods for interface modeling in damaged masonry. Advances in Engineering Software 46, 35-42, Cited By (since 1996).

Rizzoni, R., Lebon, F., 2012. Asymptotic analysis of an adhesive joint with mismatch strain. European Journal of Mechanics - A/Solids 36, 1-8. 
Schmidt, P., 2008. Modelling of adhesively bonded joints by an asymptotic method. International Journal of Engineering Science 46, 1291-1324.

Serpilli, M., Lenci, S., 2008. Limit models in the analysis of three different layered elastic strips. European Journal of Mechanics - A/Solids 27, $247-268$.

Serpilli, M., Lenci, S., 2012. Asymptotic modelling of the linear dynamics of laminated beams. International Journal of Solids and Structures 49, 1147-1157.

Zaittouni, F., Lebon, F., Licht, C., 2002. Etude théorique et numérique du comportement d'un assemblage de plaques. Comptes Rendus de l'Académie des Sciences - Series IIB - Mechanics 330, 359-364 